\title{
REVIEW
}

\section{Oral candidiasis}

\section{A Akpan, R Morgan}

Postgrad Med J 2002;78:455-459

Oral candidiasis is a common opportunistic infection of the oral cavity caused by an overgrowth of Candida species, the commonest being Candida albicans. The incidence varies depending on age and certain predisposing factors. There are three broad groupings consisting of acute candidiasis, chronic candidiasis, and angular cheilitis. Risk factors include impaired salivary gland function, drugs, dentures, high carbohydrate diet, and extremes of life, smoking, diabetes mellitus, Cushing's syndrome, malignancies, and immunosuppressive conditions. Management involves taking a history, an examination, and appropriate antifungal treatment with a few requiring samples to be taken for laboratory analysis. In certain high risk groups antifungal prophylaxis reduces the incidence and severity of infections. The prognosis is good in the great majority of cases.

See end of article for authors' affiliations

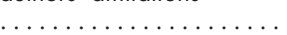

Correspondence to: Dr A Akpan, Arrowe Park Hospital NHS Trust, Upton, Wirral CH49 5PE, UK: asang@doctors.org.uk

Submitted

14 November 2001

Accepted 11 March 2002
O ral candidiasis is an opportunistic infection of the oral cavity. It is common and underdiagnosed among the elderly, particularly in those who wear dentures and in many cases is avoidable with a good mouth care regimen. It can also be a mark of systemic disease, such as diabetes mellitus and is a common problem among the immunocompromised. Oral candidiasis is caused by an overgrowth or infection of the oral cavity by a yeast-like fungus, candida. ${ }^{12}$ The important ones are $C$ albicans (the commonest; see fig 1), C tropicalis, $C$ glabrata, $C$ pseudotropicalis, C guillierimondii, C krusei, C lusitaniae, C parapsilosis, and C stellatoidea. $C$ albicans, $C$ glabrata, and $C$ tropicalis represent more than $80 \%$ of isolates from clinical infection. ${ }^{3}$ Oral candidiasis is the most common human fungal infection ${ }^{45}$ especially in early and later life. In the general population, carriage rates have been reported to range from $20 \%$ to $75 \%{ }^{4}$ without any symptoms. The incidence of $C$ albicans isolated from the oral cavity has been reported to be $45 \%$ in neonates, ${ }^{6} 45 \%-65 \%$ of healthy children, ${ }^{7} 30 \%-45 \%$ of healthy adults, ${ }^{8}$, $50 \%-65 \%$ of people who wear removable dentures, ${ }^{9} 65 \%-88 \%$ in those residing in acute and long term care facilities, ${ }^{9-12} 90 \%$ of patients with acute leukaemia undergoing chemotherapy, $^{13}$ and $95 \%$ of patients with HIV. ${ }^{14} \mathrm{C}$ albicans is a normal commensal of the mouth and generally causes no problems in healthy people. Overgrowth of candida, however, can lead to local discomfort, an altered taste sensation, dysphagia from oesophageal overgrowth resulting in poor nutrition, slow recovery, and prolonged hospital stay. In immunocompromised patients, infection can spread through the bloodstream or upper gastrointestinal tract leading to severe infection with significant morbidity and mortality. Systemic candidiasis carries a mortality rate of $71 \%$ to $79 \% .^{15}$

It is important for all physicians looking after older patients to be aware of the risk factors, diagnosis, and treatment of oral candidiasis. In a recent study $30 \%$ of doctors said they would prescribe nystatin for oral candidiasis on the request of nursing staff without examination of the oral cavity. ${ }^{16}$ This is unfortunate as other pathology may be missed, the diagnosis may be incorrect, and failure to address risk factors may lead to recurrence of the candidiasis.

\section{CLASSIFICATION}

There are a number of different types of oropharyngeal candidiasis including acute pseudomembranous, acute atrophic, chronic hyperplastic, chronic atrophic, median rhomboid glossitis, and angular cheilitis. ${ }^{17}$ The most discrete lesion represents conversion from benign colonisation to pathological overgrowth.

Pseudomembranous candidiasis (thrush) is characterised by extensive white pseudomembranes consisting of desquamated epithelial cells, fibrin, and fungal hyphae (see fig 2). These white patches occur on the surface of the labial and buccal mucosa, hard and soft palate, tongue, periodontal tissues, and oropharynx. The membrane can usually be scraped off with a swab to expose an underlying erythematous mucosa. Diagnosis is usually straightforward as it is easily seen and is one of the commonest forms of oropharyngeal candidiasis accounting for almost a third..$^{18}$ Diagnosis can be confirmed microbiologically either by staining a smear from the affected area or by culturing a swab from an oral rinse. Predisposing factors include extremes of age, diabetes mellitus, patients who have HIV/AIDS or leukaemia, those using steroid aerosol inhalers, broad spectrum antibiotics, and psychotropic drugs, and patients who are terminally ill. Other conditions that can give rise to white patches in the mouth are lichen planus, squamous cell carcinoma, lichenoid reaction, and leukoplakia.

Acute atrophic candidiasis is usually associated with a burning sensation in the mouth or on the

\section{Box 1: Introduction}

- Oral candidiasis is the commonest human fungal infection.

- Untreated, this can lead to poor nutrition and prolonged recovery.

- In extreme cases can be fatal when it becomes disseminated. 


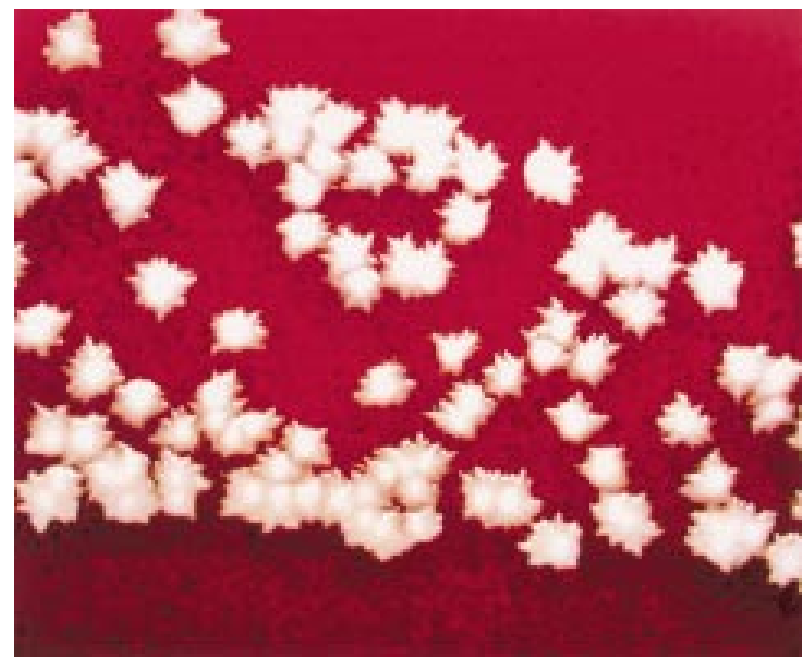

Figure 1 Candida albicans as seen under light microscopy (courtesy of Dr Cunnliffe, Consultant Microbiologist, Wirral NHS Trust).

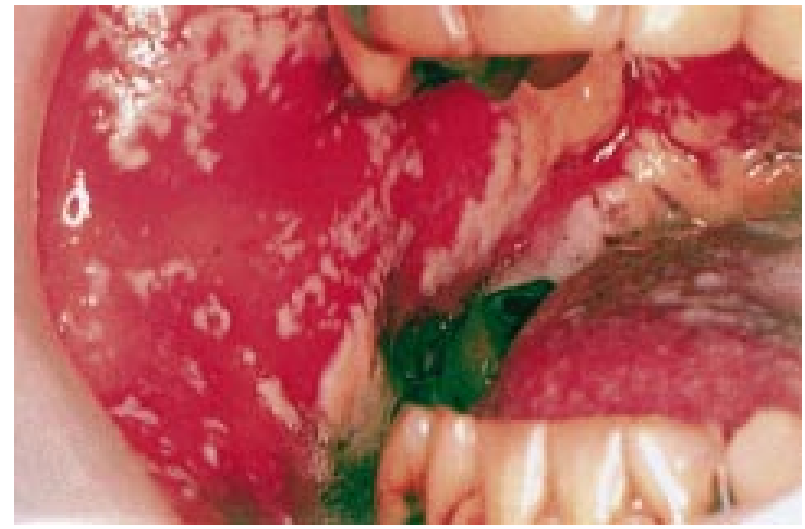

Figure 2 Acute pseudomembanous candidiasis.

tongue. The tongue may be bright red similar to that seen with a low serum B12, low folate, and low ferritin. Diagnosis may be difficult but should be considered in the differential diagnosis of a sore tongue especially in a frail older patient with dentures who has received antibiotic therapy or who is on inhaled steroids. A swab from the tongue/buccal mucosa may help diagnosis.

Chronic hyperplastic candidiasis characteristically occurs on the buccal mucosa or lateral border of the tongue as speckled or homogenous white lesions (see fig 3). The lesions usually occur on the buccal mucosa or lateral borders of the tongue. There is an association with smoking ${ }^{19}$ and complete resolution appears to be dependent on cessation of smoking. This condition can progress to severe dysplasia or malignancy and is sometimes referred to as candidal leukoplakia. Candida spp are not always isolated from lesions of oral leukoplakia and it has been suggested that the finding of Candida spp in these premalignant lesions is a complicating factor rather than a causative one. ${ }^{20}$ This condition may be confused with lichen planus, pemphigoid/pemphigus, and squamous cell carcinoma.

Chronic atrophic candidiasis also known as "denture stomatitis" is characterised by localised chronic erythema of tissues covered by dentures. Lesions usually occur on the palate and upper jaw but may also affect mandibular tissue. Diagnosis requires removal of dentures and careful inspection; swabs may be taken for confirmation. It is quite common with incidence rates of up to $65 \%$ reported.

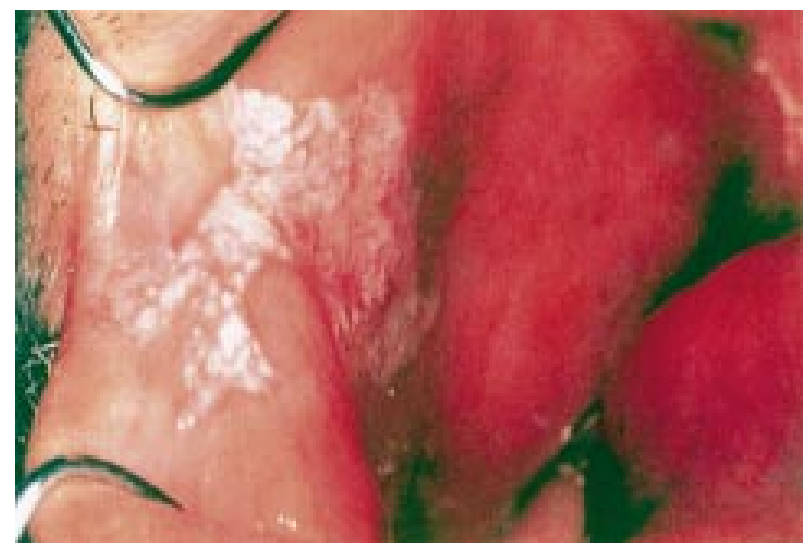

Figure 3 Chronic hyperplastic candidiasis.

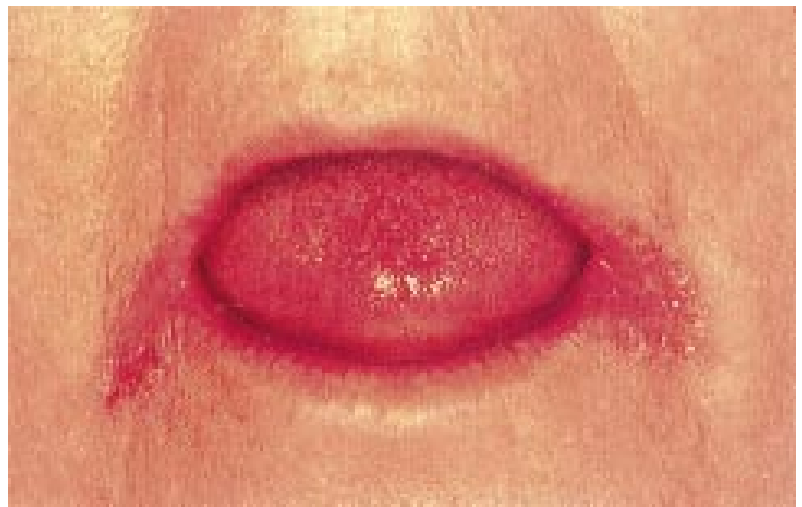

Figure 4 Angular cheilitis.

Median rhomboid glossitis is a chronic symmetrical area on the tongue anterior to the circumvallate papillae. It is made up of atrophic filiform papillae. Biopsy of this area usually yields candida $^{21}$ in over $85 \%$ of cases. It tends to be associated with smoking and the use of inhaled steroids.

Angular cheilitis is an erythematous fissuring at one or both corners of the mouth (see fig 4), and is usually associated with an intraoral candidal infection. Other organisms implicated are staphylococci and streptococci. In the case of staphylococci the reservoir is usually the anterior region of the nostrils and spread to the angles of the mouth has been confirmed by phage typing. ${ }^{22}{ }^{23}$ Facial wrinkling at the corners of the mouth and along the nasolabial fold especially in older people leads to a chronically moist environment that predisposes to this lesion. ${ }^{24}$ This wrinkling is worse in long term denture wearers because there is resorption of bone on which the dentures rest leading to a reduction in height of the lower face when the mouth is closed. ${ }^{25}$ Other factors implicated in the aetiology of this condition are iron deficiency anaemia and vitamin B12 deficiency.

\section{RISK FACTORS}

\section{(1) Pathogen}

Candida is a fungus and was first isolated in 1844 from the sputum of a tuberculous patient. ${ }^{26}$ Like other fungi, they are non-photosynthetic, eukaryotic organisms with a cell wall that lies external to the plasma membrane. There is a nuclear pore complex within the nuclear membrane. The plasma membrane contains large quantities of sterols, usually ergosterol. Apart from a few exceptions, the macroscopic and microscopic cultural characteristics of the different candida species are similar. They can metabolise glucose under both aerobic and anaerobic conditions. Temperature influences 


\section{Box 2: Classification}

Oral candidiasis can be classified as follows:

\section{Acute candidiasis}

- Acute pseudomembranous candidiasis (thrush).

- Acute atrophic (erythematous) candidiasis.

2. Chronic candidiasis

- Chronic hyperplastic candidiasis (candidal leukoplakia)

- Denture induced candidiasis (chronic atrophic (erythematous) candidiasis).

- Median rhomboid glossitis.

\section{Angular cheilitis (stomatitis)}

\section{Box 3: Risk factors for oropharyngeal candidiasis}

- Pathogen has peculiar properties that increase its infectivity rate in the right environment.

- Host factors could be local and/or systemic.

- Local factors include wearing dentures, impaired salivary gland function, inhaled steroids, and oral cancer

- Systemic factors include extremes of age, smoking, diabetes mellitus, Cushing's syndrome, immunosuppression, malignancies, nutritional deficiencies, and antibiotics.

their growth with higher temperatures such as $37^{\circ} \mathrm{C}$ that are present in their potential host, promoting the growth of pseudohyphae. They have been isolated from animals and environmental sources. They can be found on or in the human body with the gastrointestinal tract, the vagina, and skin being the most common sites and $C$ albicans being the commonest species isolated from these sites. They require environmental sources of fixed carbon for their growth. Filamentous growth and apical extension of the filament and formation of lateral branches are seen with hyphae and mycelium, and single cell division is associated with yeasts. ${ }^{27}$

Several studies have demonstrated that infection with candida is associated with certain pathogenic variables. Adhesion of candida to epithelial cell walls, an important step in initiation of infection, is promoted by certain fungal cell wall components such as mannose, C3d receptors, mannoprotein, and saccharins. ${ }^{22}{ }^{28-30}$ The degree of hydrophobicity ${ }^{31}$ and ability to bind to host fibronectin ${ }^{32}$ has also been reported to be important in the initial stages of infection. Other factors implicated are germ tube formation, presence of mycelia, persistence within epithelial cells, endotoxins, induction of tumour necrosis factor, and proteinases. ${ }^{33-38}$ Phenotypic switching which is the ability of certain strains of $C$ albicans to change between different morphologic phenotypes has also been implicated. ${ }^{39}$

\section{(2) Host}

\section{Local factors}

Impaired salivary gland function can predispose to oral candidiasis. ${ }^{10}$ Secretion of saliva causes a dilutional effect and removes organisms from the mucosa. Antimicrobial proteins in the saliva such as lactoferrin, sialoperoxidase, lysozyme, histidine-rich polypeptides, and specific anticandida antibodies, interact with the oral mucosa and prevent overgrowth of candida. Therefore conditions such as Sjögren's syndrome, radiotherapy of the head and neck, or drugs that reduce salivary secretions can lead to an increased risk of oral candidiasis.

Drugs such as inhaled steroids have been shown to increase the risk of oral candidiasis ${ }^{41}$ by possibly suppressing cellular immunity and phagocytosis. The local mucosal immunity reverts to normal on discontinuation of the inhaled steroids. ${ }^{42}$
Dentures predispose to infection with candida in as many as $65 \%$ of elderly people wearing full upper dentures. ${ }^{20}$ Wearing of dentures produces a microenvironment conducive to the growth of candida with low oxygen, low $\mathrm{pH}$, and an anaerobic environment. This may be due to enhanced adherence of Candida spp to acrylic, reduced saliva flow under the surfaces of the denture fittings, improperly fitted dentures, or poor oral hygiene. ${ }^{12}$

Other factors are oral cancer/leukoplakia and a high carbohydrate diet. Growth of candida in saliva is enhanced by the presence of glucose and its adherence to oral epithelial cells is enhanced by a high carbohydrate diet. ${ }^{43}$

\section{Systemic factors}

Extremes of life predispose to infection because of reduced immunity. ${ }^{2}$

Drugs such as broad spectrum antibiotics alter the local oral flora creating a suitable environment for candida to proliferate. ${ }^{44}$ The normal oral flora is restored once the antibiotics are discontinued. Immunosuppressive drugs such as the antineoplastic agents have been shown in several studies to predispose to oral candidiasis by altering the oral flora, disrupting the mucosal surface and altering the character of the saliva. ${ }^{13} 4546$

Other factors are smoking, diabetes, Cushing's syndrome, immunosuppressive conditions such as HIV infection, malignancies such as leukaemia, and nutritional deficienciesvitamin $\mathrm{B}$ deficiencies have been particularly implicated. Ninane found that $15 \%-60 \%$ of people with malignancies will develop oral candidiasis while they are immunosuppressed. ${ }^{47}$ In those with HIV infection rates of between $7 \%$ to $48 \%$ have been quoted and more than $90 \%$ has been reported in those with advanced disease. Relapse rates are between $30 \%$ and $50 \%$ on completion of antifungal treatment in severe immunosuppression. ${ }^{48}$

\section{MANAGEMENT}

Taking a history followed by a thorough examination of the mouth, looking at the soft and hard palate, and examining the buccal mucosa in those wearing dentures after they have been removed are usually good starting points. Predisposing factors are identified as mentioned above and resolved if possible, and the type, severity, and chronicity of the infection are assessed.

The right diagnosis is usually made on finding the characteristic lesion, ruling out other possibilities, and the response to antifungal treatment. Acute pseudomembranous and chronic atrophic candidiasis can be treated based on clinical features but culture and sensitivity testing should be undertaken if initial therapy is unsuccessful. Imprint cultures, ${ }^{5}$ where sterile foam pads dipped in Sabouraud's broth are placed for 30 seconds on the lesion and then placed on Sabouraud's agar containing chloramphenicol for an hour after which they are incubated, have also been used for identification of Candida spp. Acute atrophic and chronic hyperplastic forms may mimic other lesions and a biopsy is recommended in addition to empirical therapy to rule out more serious lesions such as squamous cell carcinoma.

Oral hygiene and topical antifungals are usually adequate for uncomplicated oral candidiasis.

Oral hygiene involves cleaning the teeth, buccal cavity, tongue, and dentures, if present, daily. Dentures should be cleaned and disinfected daily and left out overnight or for at least six hours daily. The dentures should be soaked in a denture cleaning solution such as chlorhexidine as this is more effective in eliminating candida than brushing. ${ }^{49}$ This is because dentures have irregular and porous surfaces to which candida easily adheres and brushing alone cannot remove them. When rinsing the mouth with the topical antifungal, dentures should be removed to allow contact between the mucosa and the antifungal. The patient should ensure that the 
whole mucosa is coated with the antifungal and held in the mouth for a few minutes. The incorporation of an antifungal with a denture liner has been recommended for patients with dentures who find it difficult to hold the antifungal in their mouth for a few minutes. Also the mucosal surface should be brushed regularly with a soft brush. After disinfection, dentures should be allowed to air dry as this also kills adherent candida on dentures. ${ }^{50}$ Chlorhexidine can discolour both dentures and natural dentition if not removed adequately after disinfection. A referral to a dentist might be necessary for those with poorly fitting dentures as these predispose to infection by breaking down the epithelial barrier. Other denture cleaning methods not routinely used but shown to be effective are ultrasonic cleaning tanks with a suitable solution. ${ }^{51}$

Regular oral and dental hygiene with periodic oral examination will prevent most cases of oral candidiasis in those with dentures. Combining nystatin with chlorhexidine digluconate, an antiseptic used to disinfect dentures, inactivates both drugs $s^{52}{ }^{53}$ therefore this combination should not be used. The dentures should be removed each time the mouth is rinsed with the oral antifungal preparation in established cases of denture stomatitis and the dentures soaked in chlorhexidine before putting them back in the mouth.

Topical antifungal therapy is the recommended first line treatment for uncomplicated oral candidiasis and where systemic treatment is needed topical therapy should continue as this reduces the dose and duration of systemic treatment required.$^{54}$ The systemic adverse effects and drug interactions that occur with the systemic agents do not occur with topical agents. ${ }^{44}$ Treatment in the early part of the 20th century was with gentian violet, an aniline dye, but because of resistance developing and side effects, such as staining of the oral mucosa, it was replaced by a polyene antibiotic, nystatin, discovered in 1951 and amphotericin B, discovered in 1956. They act by binding to sterols in the cell membrane of fungi, and, altering cell membrane permeability. ${ }^{55} 56$

Nystatin and amphotericin are not absorbed from the gastrointestinal tract and are used by local application in the mouth. Miconazole, an imidazole, can be used as a local application in the mouth but its use in this way is limited because of potential side effects such as vomiting and diarrhoea. Other drugs belonging to this class are clotrimazole and ketoconazole. Nystatin is the most widely used topical agent for the treatment of oral candidiasis. ${ }^{12}$ It is available as an oral rinse, pastille, and suspension. It should be used as a rinse four times a day for two weeks. It can cause nausea, vomiting, and diarrhoea. The oral rinse contains sucrose and is useful in edentulous patients and those with xerostomia such as patients receiving radiotherapy and those with HIV infection. ${ }^{57}$ Clotrimazole troche can be an alternative for those patients who find nystatin suspensions unpalatable.

Systemic antifungal therapy in oral candidiasis is appropriate in patients intolerant of or refractory to topical treatment and those at high risk of developing systemic infections. ${ }^{54}$

Both nystatin oral rinses and clotrimazole troches have a high sucrose content and if tooth decay is a concern or the oral candidiasis is complicated by diabetes, steroid use or an immunocompromised state, triazoles which include fluconazole or itraconazole once per day has been found to be effective in these cases. ${ }^{58}$ Ketoconazole is also as effective as fluconazole and itraconazole but its use in elderly patients is not recommended due to drug interactions and side effects, which include hepatotoxicity.

Fluconazole is a potent and selective inhibitor of fungal enzymes involved in the synthesis of ergosterol, an important constituent of the plasma cell membrane. It therefore disrupts cell wall formation leading to leakage of cellular contents and cell death. It is well absorbed by the gastrointestinal tract and the plasma levels are over $90 \%$ of the levels achieved with intravenous administration and the levels in saliva and

\section{Box 4: Management}

- Diagnosis is usually made on clinical grounds with laboratory testing to exclude potentially other serious oral lesions especially squamous cell carcinoma.

- Predisposing factors should be treated or eliminated where feasible.

- Good oral hygiene is important.

- Topical antifungals given for two weeks are usually effective.

- Systemic antifungals should be given in certain circumstances.

sputum are also similar to that in the plasma. It is preferred, as it does not have the same hepatotoxicity as the imidazoles. It is now listed in the dental practitioners' formulary as well as the British National Formulary and is therefore widely used both in dental as well as medical practice but there are problems with resistance.

Itraconazole has a wider spectrum of activity than fluconazole and is therefore valuable in salvage treatment of the immunocompromised patients with fluconazole resistant candidosis. Increasing resistance to antifungals has become increasingly common since the introduction of fluconazole especially in patients with advanced HIV disease, and recurrent and long term treatment. ${ }^{5960}$

Angular cheilitis is treated with antifungal steroid creams and ointments and any concurrent intraoral lesion is also treated at the same time and dietary deficiencies should be excluded and treated if found.

Failure to respond to therapy especially in chronic atrophic candidiasis is usually due to non-compliance with treatment.

Prophylaxis with antifungal agents reduces the incidence of oral candidiasis in patients with cancer undergoing treatment ${ }^{61}$ and fluconazole has been found to be more effective than topical polyenes. ${ }^{62}$

Prophylaxis on either a daily or weekly basis with antifungals reduces the incidence of oral candidiasis in patients with HIV with the reductions being most marked in those with low CD4 counts and recurrent oral candidiasis. ${ }^{63-66}$ The use of a chlorhexidine rinse only in bone marrow transplant patients as prophylaxis was found to be very effective. ${ }^{67}$

\section{PROGNOSIS}

The prognosis is good for oral candidiasis with appropriate and effective treatment. Relapse when it occurs is more often than not due to poor compliance with therapy, failure to remove and clean dentures appropriately, or inability to resolve the underlying/predisposing factors to the infection.

\section{Authors' affiliations}

A Akpan, R Morgan, Arrowe Park Hospital NHS Trust, Upton, Wirral, UK

\section{REFERENCES}

1 Epstein JB. Antifungal therapy in oropharyngeal mycotic infections. Oral Surg Oral Med Oral Pathol 1990:69:32-41.

2 Guida RA. Candidiasis of the oropharynx and oesophagus. Ear Nose Throat J 1988;67:832-40

3 Odds FC. Candida and candidiasis. 2nd Ed. London: Bailliere Tindall, 1988.

4 Ghannoum MA, Radwan SS. Candida adherence to epithelial cells. Boca Raton, FL: CRC Press, 1990.

5 Abu-Elteen KH, Abu-Alteen RM. The prevalence of candida albicans populations in the mouths of complete denture wearers. New Microbiol 1998;21:41-8.

6 Manning DJ, Coughlin RP, Poskit EM. Candida in mouth or on dummy? Arch Dis Child 1985;60:381-2.

7 Berdicevsky I, Ben-Aryeh H, Sazargel R, et al. Oral candida in children Oral Surg Oral Med Oral Pathol 1980;57:37-40

8 Lucas VS. Association of psychotropic drugs, prevalence of denture-related stomatitis and oral candidosis. Community Dent Oral Epidemiol 1993;21:313-16. 
9 Arendorf TM, Walker DM. The prevalence and intra-oral distribution of Candida albicans in man. Arch Oral Biol 1980;25:1-10.

10 Aldred MJ, Addy M, Bagg J, et al. Oral health in the terminally ill: a cross sectional pilot survey. Spec Care Dentist 1991;11:59-62.

11 Cumming CG, Wight C, Blackwell CL, et al. Denture stomatitis in the elderly. Oral Microbiol Immunol 1990;5:82-5.

12 Holbrook WP, Hiorleifsdottir DV. Occurrence of oral Candida albicans and other yeast-like fungi in edentulous patients in geriatric units in Iceland. Gerodontics 1986;2;153-6.

13 Rodu B, Carpenter JT, Jones MR. The pathogenesis and clinical significance of cytologically detectable oral candida in acute leukaemia. Cancer 1988;62:2042-6.

14 Dupont B, Graybill JR, Armstrong D, et al. Fungal infections in AIDS patients. J Med Vet Mycol 1992; 30(suppl 1):19-28.

15 Fraser VJ, Jones M, Dunkel J, et al. Candidemia in a tertiary care hospital: epidemiology, risk factors, and predictors of mortality. Clin Infect Dis 1992;15:414-21.

16 Morgan R, Tsang J, Harrington N, et al. Survey of hospital doctor's attitudes and knowledge of oral conditions in older patients. Postgrad Med J 2001;77:392-4

17 Lewis MAO, Lamey P-J. Clinical oral medicine. Oxford: Butterworth-Heinemann, 1995.

18 Samaranayake LP. Nutritional factors and oral candidiasis. J Ora Pathol 1986;15:61-5.

19 Silverman S, Luangiarmekorn L, Greenspan D. Occurrence of oral candida in irradiated head and neck cancer patients. J Oral Med 1984;39: 194-6.

20 Dreizen S. Oral candidiasis. Am J Med 1984;30:28-33.

21 Budzt-Jorgenson E. Etiology, pathogenesis, therapy and prophylaxis of oral yeast infections. Acta Odontol Scand 1990:48:61-9.

22 Kanbe T, Li R-K, Wadsworth E, et al. Evidence for expression of the C3d receptor of candida albicans in-vitro and in-vivo obtained by immunoflourescence and immunoelectron microscopy. Infect Immun 1991;59:1832.

23 MacFarlane TW, Helnarska SJ. The microbiology of angular cheilitis. $\mathrm{Br}$ Dent J 1968;140:403-6.

24 Shay K, Truhlar MR, Renner RP. Oropharyngeal candidosis in the older patient. J Am Geriatr Soc 1997;45:863-70.

25 Penhall B. Preventive measures to control further bone loss and soft tissue damage in denture wearing. Aust Dent J 1980;25:319-24.

26 Mandell GL, Bennett JE, Dolin R. Anti-fungal agents. Principles and practice of infectious diseases. 4th Ed. New York: Churchill Livingstone, 1994: 401-10

27 Lehmann PF. Fungal structure and morphology. Medical Mycology 1998;4:57-8.

28 Brassart D, Woltz A, Golliard M, et al. In-vitro inhibition of adhesion of Candida albicans clinical isolates to human buccal epithelial cells by Fuc $\alpha 1 \rightarrow 2$ Gal $\beta$-bearing complex carbohydrates. Infect Immun 1991;59:1605

29 Channoum MA, Burns GR, Elteen A, et al. Experimental evidence for the role of lipids in adherence of candida spp to human buccal epithelia cells. Infect Immun 1986;54:189.

30 Douglas LJ. Surface composition and adhesion of Candida albicans. Bio Soc Trans 1985:13:982.

31 Hazen KC, Brawner DL, Riesselman MH, et al. Differential adherence of hydrophobic and hydrophilic Candida albicans yeast cells to mouse tissues. Infect Immun 1991;59:907

32 Klotz SA, Smith RL. A fibronectin receptor on Candida albicans mediates adherance of the fungus to extracellular matrix. J Infect Dis $1991 ; 163: 604$

33 Sobel JD, Muller G, Buckley HR. Critical role of germ tube formation in the pathogenesis of candidal vaginitis. Infect Immun 1984;44:576.

34 Saltarelli CG, Gentile KA, Mancuso SC. Lethality of candidal strains as influenced by the host. Can J Microbiol 1975;21:648.

35 Smith CB. Candidiasis: pathogenesis, host resistance, and predisposing factors. New York: Raven Press, 1985

36 Cutler JE, Friedman L, Milner KC. Biological and chemical characteristics of toxic substances from Candida albicans. Infect Immun 1972;6:616.

37 Riipi L, Carlson E. Tumour necrosis factor (9TNF) is induced in mice by Candida albicans: role of TNF in fibrinogen increase. Infect Immun 1990;58:2750

38 Kwon-Chung KJ, Lehman D, Good C, et al. Genetic evidence for role of extracellular proteinase in virulence of Candida albicans. Infect Immun 1985:49:571.

39 Slutsky B, Buffo J, Soll DR. High frequency switching of colony morphology in Candida albicans. Science 1985;230:666.

40 Peterson DE. Oral candidiasis. Clin Geriatr Med 1992;8:513-27.
41 Milne LJ, Crompton GK. Beclomethasone dipropionate and oropharyngeal candidiasis. BM 1974;iii:797-8.

42 Garber GE. Treatment of oral candida mucositis infections. Drugs 1994; 47:734-40

43 Ohman SC, Jontell M. Treatment of angular cheilitis: the significance of microbial analysis, antimicrobial treatment, and interfering factors. Acta Odontol Scand 1988;46:267-72.

44 Epstein JB, Truelove EL, Izutzu KL. Oral candidiasis: pathogenesis and host defense. Rev Infect Dis 1984;6:96-106.

45 Francis $\mathbf{P}$, Walsh TJ. Current approaches to the management of fungal infections in cancer patients. Oncology 1992;6:81-92.

46 Bergman OJ. Alterations in oral microflora and pathogenesis of acute oral infections during remission-induction therapy in patients with acute myeloid leukaemia. Scand J Infect Dis 1991;23:355-66.

47 Ninane JA. Multicentre study of fluconazole versus oral polyenes in the prevention of fungal infection in children with haematological or oncological malignancies. Multicentre study group. Eur J Clin Microbiol Infect Dis 1994;13:330-7

48 Phillips P, Zemcov J, Mahmood W, et al. Itraconazole cyclodextrin solution for fluconazole refractory oropharyngeal candidiasis in AIDS: correlation of clinical response with in vitro susceptibility. AIDS 1996;10:1369-76.

49 Odman PA. The effectiveness of an enzyme containing denture cleaner. Quintessence Int 1992;23:187-90.

50 Stafford GD, Arendorf GD, Huggett R. The effect of overnight drying and water immersion on candidal colonisation and properties of complete dentures. J Dent 1986;14:52-6.

51 Gwinnet AJ, Caputo L. The effectiveness of ultrasonic denture cleaning: a scanning electron microscopy study. J Prosthet Dent 1983;50:20-5.

52 Barkvoll P, Attramadal A. Effect of nystatin and chlorhexidine digluconate on Candida albicans. Oral Surg Oral Med Oral Pathol 1989;67:279-81.

53 Barkvoll P, Hurlen B.Conventional treatment of oral candidiasis. New aspects. Nor Tannlaegeforen Tid 1989;99:116-19.

54 Epstein JB, Polsky B. Oropharyngeal candidiasis: a review of its clinical spectrum and current therapies. Clin Ther 1998;20:40-57.

55 Gupta AK, Sauder DN, Shear NH. Antifungal agents; an overview. Part 1. J Am Acad Dermatol 1994;30:677-98.

56 Bennett JE. Antimicrobial agents, antifungal agents. In: Gilman AG, Rall TW, Nies AS, et al, eds. Goodman and Gilman's the pharmacological basis of therapeutics. 8th Ed. New York: Pergamon Press, 1990: 1165-81.

57 Epstein JB, Frelich MM, Le ND. Risk factors for oropharyngeal candidiasis in patients who receive radiation therapy for malignant conditions of the head and neck. Oral Surg Oral Med Oral Pathol 1993;76:169-74.

58 Blatchford NR. Treatment of oral candidiasis with itraconazole: a review. J Am Acad Dermatol 1990;23:565-7.

59 Heine GS, Stevens DS, Greenspan D. Fluconazole-resistant candida in AIDS patients. Oral Surg Oral Med Oral Pathol 1993:76:711-15.

60 Rex JH, Rinaldi MG, Pfaller MA. Resistance of candida species to fluconazole. Antimicrob Agents Chemother 1995;39:1-8.

61 Clarkson JE, Worthington HV, Eden OB. Prevention of oral mucositis or oral candidiasis for patients with cancer receiving chemotherapy (excluding head and neck cancer). The Cochrane Library, Issue 4,2000. Oxford.

62 Lumbreras C, Cuervas-Mons V, Jara $P$, et al. Randomised trial of fluconazole versus nystatin for the prophylaxis of candida infection following liver transplantation. J Infect Dis 1996;174:583-8.

63 Schuman P, Capps L, Peng G, et al. Weekly fluconazole for the prevention of mucosal candidiasis in women with HIV infection. A randomised, double-blind, placebo controlled trial. Terry Beirn community programs for clinical research on AIDS. Ann Intern Med 1997; 126:689-96.

64 MacPhail LA, Hilton JF, Dodd CL, et al. Prophylaxis with nystatin pastilles for HIV-associated oral candidiasis. J Acquir Immune Defic Syndr 1996; 12:470-6

65 Powderly WG, Finklestein DM, Feinberg J, et al. A randomised trial comparing fluconazole with cotrimazole troches for the prevention of fungal infection in patients with advanced human immunodeficiency virus infection. N Engl J Med 1995;332:700-5

66 Smith D, Midgley J, Gazzard B. A randomised, double-blind study of itraconazole versus placebo in the treatment and prevention of oral or oesophageal candidiasis in patients with HIV infection. Int J Clin Pract 1999:53:349-52

67 Ferretti GA, Ash RC, Brown AT, et al. Chlorhexidine for prophylaxis against oral infections and associated complications in patients receiving bone marrow transplants. Am Dent Assoc 1987;114:461-7. 